\title{
Erratum: Longer hospital stay is associated with high rates of tuberculosis-related morbidity and mortality within 12 months after discharge in a referral hospital in Sub-Saharan Africa
}

\author{
Nicola M Zetola ${ }^{1,2,3^{*}}$, Nenad Macesic ${ }^{4}$, Sanghyuk S Shin ${ }^{5}$, Alexandra Peloso ${ }^{6}$, Ronald Ncube ${ }^{7}$, Jeffrey D Klausner ${ }^{5}$, \\ Chawangwa Modongo ${ }^{1,3}$ and Ronald G Collman ${ }^{8}$
}

\section{Erratum}

Following publication of the original article [1] we became aware of the following errors which we wish to correct. These corrections have no impact over the study results, their interpretation or conclusions.

\section{Authors and affiliations}

The correct list of authors and their respective affiliations is the following:

Nicola M Zetola ${ }^{1,2,3}$, Nenad Macesic ${ }^{4}$, Sanghyuk S. $\mathrm{Shin}^{5}$, Alexandra Peloso ${ }^{6}$, Ronald $\mathrm{Ncube}^{7}$, Jeffrey D Klausner $^{5}$, Chawangwa Modongo ${ }^{1,3}$, Ronald G Collman ${ }^{8}$

1. Division of Infectious Disease, University of Pennsylvania, Philadelphia, Pennsylvania;

2. University of Botswana Medical School, University of Botswana, Gaborone, Botswana;

3. Botswana-UPenn Partnership, Gaborone, Botswana;

4. Division of Infectious Diseases, University of Melbourne, Melbourne, Australia;

5. University of California, Los Angeles, Los Angeles, California;

6. University of Western Ontario; School of Medicine, Botswana,

7. Botswana National TB Program, Gaborone, Botswana

8. Division of Pulmonary and Critical Care Medicine, University of Pennsylvania, Philadelphia, Pennsylvania

\footnotetext{
* Correspondence: nzetola@gmail.com

${ }^{1}$ Division of Infectious Disease, University of Pennsylvania, Philadelphia, PA, USA

${ }^{2}$ University of Botswana Medical School, University of Botswana, Gaborone, Botswana

Full list of author information is available at the end of the article
}

\section{Abstract}

1. The following text "Eventually, 916 (83.7\%) patients were discharged and followed for one year after it. Of these, 51 (5.6\%) were diagnosed with PTB during the year of follow up (annual TB rate of 3,712 cases per 100,000 person per year)" should be replaced with "Of the 896 (83.7\%) discharged patients, 41 (4.6\%) were diagnosed with TB during the year of follow-up. Overall, 123/896 (14\%) patients died during the follow up period, of whom 26/123 (21\%) died from TB."

Table 3

2. The row 'No TB' (last row [row 18]) should read 879, not 979.

3. The column 'No. of Patients' (column 2) should also read 879 , not 979 .

4. The column entitled "TB incidence per 100 persons per year of follow up" (column 9) should be entitled "TB incidence during the one-year follow up".

5. There is a typo in the column "Average TB exposure index" (column 7), row "HIV status positive" (row 7). The decimal number is missing. It reads "12." and should be " 12.3 ".

6. In the last 2 columns, the frequencies under the subheading "CD4 cell count" and "Diagnosis of PTB during admission" have been switched. That is, the numbers currently under the "TB-related mortality" column (the last column; $\mathrm{n}=4,4,10,9,9,7$ ) belong to the "Overall Mortality" column (second to last column; $\mathrm{n}=2,3,4,5,5,2)$ and vice versa. 
7. Same as above, the numbers for "Diagnosis of TB at admission" ( $n=17,5)$ should be in the last column ("TB-related mortality") and the numbers 26, 31 should be in the "Overall Mortality" (second to last) column.

8. The table headings of the last 2 columns should reflect that these are (n)'s, not (\%)'s.

\section{Table 4}

9. The sample size for the column entitled "Models including participants who were diagnosed with TB during their inpatient admission" is 896 , not 916 .

\section{In additional file 3}

10. The sample size should be noted as $n=896$.

11. The column heading "Incident TB during 1-year follow-up" and "Death during 1-year of follow-up" should indicate that the reported measures are unadjusted odds ratios.

\section{Author details}

${ }^{1}$ Division of Infectious Disease, University of Pennsylvania, Philadelphia, PA, USA. ${ }^{2}$ University of Botswana Medical School, University of Botswana, Gaborone, Botswana. 'Botswana-UPenn Partnership, Gaborone, Botswana. ${ }^{4}$ Division of Infectious Diseases, University of Melbourne, Melbourne, Australia. ${ }^{5}$ School of Medicine, University of California, Los Angeles, USA. 'University of Western Ontario, School of Medicine, London, Canada.

${ }^{7}$ Botswana National TB Program, Gaborone, Botswana. ${ }^{8}$ Division of Pulmonary and Critical Care Medicine, University of Pennsylvania, Philadelphia, PA, USA.

\section{Reference}

1. Zetola NM, Macesic N, Modongo C, Shin S, Ncube R, Collman RG. BMC Infect Dis. 2014;14:409.

\section{Submit your next manuscript to BioMed Central and take full advantage of:}

- Convenient online submission

- Thorough peer review

- No space constraints or color figure charges

- Immediate publication on acceptance

- Inclusion in PubMed, CAS, Scopus and Google Scholar

- Research which is freely available for redistribution 